\section{Analys is of Endodontist Posture Utilizing Cinemetry, Surface Electromyography and Ergonomic Checklists}

Geraldo Celso da Silva Onety1, Daniel Vilela Leonel ${ }^{1}$, Paulo César Saquy ${ }^{3}$, Gabriel Pádua da Silva1', Bruno Ferreira1', Tiago Gilioli Varise'1, Luiz Gustavo de Sousa1, Edson Donizetti Verri², Selma Siéssere¹, Marisa Semprini1, Victor Rodrigues Nepomuceno ${ }^{1}$, Simone Cecilio Hallak Regalo ${ }^{1}$
'Department of Morphology, Physiology and Basic Pathology, School of Dentistry of Ribeirão Preto, USP - University of São Paulo, Ribeirão Preto, SP, Brazil ${ }^{2}$ Claretiano University Center, Batatais, SP, Brazil

${ }^{3}$ Department of Restorative Dentistry, School of Dentistry of Ribeirão Preto, USP - University of São Paulo, Ribeirão Preto, SP, Brazil

Correspondence: Profa. Dra. Simone Cecilio Hallak Regalo, Avenida do Café s/n, 14040-904 Ribeirão Preto, SP, Brasil. Tel:+55-16-3602-0281. e-mail: simone@forp.usp.br
The postural risk factors for dentists include the ease of vision in the workplace, cold, vibration and mechanical pressure in tissues, incorrect posture, functional fixity, cognitive requirements and work-related organizational and psychosocial factors. The objective was to analyze the posture of endodontists at the workplace. Eighteen right-handed endodontists aged 25 to 60 years ( $34 \pm 3)$ participated in the study. Electromyography, kinemetry, ergonomic scales (RULA and Couto's checklist) and biophotogrammetry were used to analyze the posture of endodontists during root canal treatment of the maxillary right first and second molars using rotary and manual instrumentation. The variations observed in the electromyographic activities during the performance of rotary and manual techniques suggest that the fibers of the longissimus region, anterior and medium deltoid, medium trapezium, biceps, triceps brachii, brachioradialis and short thumb abductor muscles underwent adaptations to provide more accurate functional movements. Computerized kinemetry and biophotogrammetry showed that, as far as posture is concerned, rotary technique was more demanding than the manual technique. In conclusion, the group of endodontists evaluated in this study exhibited posture disorders regardless of whether the rotary or manual technique was used.
Key Words: posture analysis, ergonomics, electromyography, rotary instrumentation.

\section{Introduction}

The postural risk factors for dentists include the ease of vision at workplace, cold, vibration and mechanical pressure on tissues, incorrect posture, skeletal muscle load, static load, functional fixity, cognitive requirements and work-related organizational and psychosocial factors. Musculoskeletal disorders developed at workplace increasingly affect the health of Brazilian workers and are the third most prevalent category of diseases. Work-related musculoskeletal disorders (WMSDs) are caused by inflexible and high intensity work cycles, repetitive movements performed at high speed, work by certain muscle groups, lack of rest periods, a high rate of productivity, and the use of ergonomically unsuitable equipment and office furniture (1).

Implementation of ergonomic principles aimed at correcting the postures is fundamental to ensure the health, safety, high performance, motivation and satisfaction of endodontists. The ergonomic features of an individual's posture and movement are determined by the activity and the workplace. When a posture or movement is adopted, many ligaments and joints are activated, and various muscles are called upon to provide the necessary strength.
Repetitive strain injury and work-related osteomuscular disturbances are among the main biomechanical risks at the workplace and their incidences have increased significantly in various health professions $(1,2)$. The most common occupational diseases among dentists include postural changes; muscular pain in the dorsal and lumbar regions as well as in legs, arms and feet; headaches; circulatory problems and varicose veins; bursitis of the shoulders and elbows; tendon inflammation; problems associated with cervical, dorsal and lumbar changes; degeneration of the cervical disc; eye fatigue; shoulder height inequality; inflammation of the tendon sheaths; hand arthritis; muscular hypertrophy in the most used limb and physiological muscle contracture $(3,4)$.

The Rapid Upper Limb Assessment (RULA) provides a rapid assessment of the load imposed on the skeletomuscular system and is a component of the overall ergonomic analysis of the performed task (4). Couto's checklist (5) is a subjective and simplified instrument used to assess the possible risks of work-related musculoskeletal disorders and tenosynovitis of the upper limbs. Electromyographic monitoring of the electrical activity of excitable membranes by measuring the motor unit action potentials is another 
effective method of analyzing posture (6). Photogrammetry is used to reconstruct the three-dimensional space from two-dimensional images produced by the recording patterns of electromagnetic waves. This method represents a non-invasive and painless way to investigate spatial measures related to the human body (7). Videogrammetry is performed using optical procedures in which measurements are made based on indirect indicators obtained from images and it also provides the kinematic parameters of movement. The use of rotational systems represents a novel technological development in endodontics, mainly because it provides a faster method for the treatment of obliterated, straight and curved molar root canals (8). Some techniques are important for the diagnosis of postural changes and muscular activity disorders.

The purpose of this study was to analyze the posture of endodontists during the chemomechanical preparation of the canal system of molars on the maxillary right hemiarch by electromyography, videogrammetry, ergonomic rating scales (RULA and Couto's Checklist) and biophotogrammetry. Two techniques were used: rotary instrumentation and manual instrumentation in the maxillary right first and second molars, respectively. The manual and rotary techniques were compared in terms of the postural adjustments made by the professionals during working hours. The study hypothesis was that the rotary technique results in less postural impairment for the endodontists.

\section{Material and Methods}

\section{Participants}

Eighteen right-handed endodontists between 25 and 60 years of age with a mean age of $34 \pm 3$ years were selected for the study. These endodontists were submitted to electromyographic, videogrammetric and biophotogrammetric analyses and evaluated by ergonomic rating scales (RULA and Couto's Checklist) while performing rotary (maxillary right second molar) and manual (maxillary right first molars) endodontic treatment techniques.

This study was approved by the Research Ethics Committee of the University of São Paulo (Protocol \#2010.1.1334.58.3). All professionals were informed about the experiment and agreed to participate by providing their informed consent according to Resolution 466/12 of the National Health Council of Brazil. All experiments were conducted in accordance with the approved guidelines.

Sample selection and the inclusion/exclusion criteria were determined as follows: all of the observed professionals were dentists and endodontic specialists with three to fifteen years of experience in rotary and manual instrumentation who had no diagnosis of postural pathologies nor of cognitive or mental problems.

\section{Research "Patient"}

A single dentist carried out all operative stages described below to avoid bias based on the initial operative procedure.

A dental simulator mannequin was used as the "patient". The mannequin was adult sized and exhibited articulation of the upper and lower limbs and neck, simulating the movements performed by a real individual. This model also had an oral cavity that included natural teeth, allowing for the replacement of the maxillary right first and second molars each time the experiment was performed, as these teeth were set in individual blocks. Eighteen maxillary right second molars and 18 maxillary right second molars were used. Prior to the experiment, the teeth had the surfaceadhered calculi removed with periodontal curettes and were polished using Robinson bristle brush with pumice paste. Then, the teeth were fixed in a wax base by the root portion and set in a block of self-cured acrylic resin obtained from a silicone matrix that had been previously prepared.

After polymerization of the acrylic resin, removing the entire pulp chamber roof and preparing a convenient form for the maxillary molar teeth made the coronal access opening. The form was triangular in shape, with the base of the triangle on the buccal side providing a complete view of the root openings. The entry of the root canal was located using a straight probe, after which the walls were straightened and all remaining carious dentin and defective restoration were removed using a high-speed Endo Z bur (Maillefer, Ballaigues, Switzerland), which enabled direct and free access to the root canals. For standardization purposes, only teeth with three openings were used. The entire procedure was carried out under copious irrigation with $1 \% \mathrm{NaOCl}$ with suction. After wax removal, canals were negotiated with a $\mathrm{K} 10$-file hand file and root canal length up to the root opening was established by direct visualization of the file tip. The working length was established $1 \mathrm{~mm}$ short of the apical foramen to avoid radiographic examination while performing the chemomechanical preparation. The procedure was completed by placing a cotton pellet inside the pulp chamber and sealing the cavity using Coltosol cement as a provisional sealing material (Vigodent S.A., Rio de Janeiro, $\mathrm{RJ}$, Brazil). Then, the blocks were placed in the maxillary right hemiarch and fixed by orthodontic acrylic resin in the palatal region to completely isolate the teeth. Finally, the dental simulator mannequin was positioned in the dental chair for the treatment procedures.

\section{Chemomechanical Preparation of the Root Canal System}

In this part of the study, the endodontic professionals were initially asked to carry out a rotary-based instrumentation technique for maxillary right first molar 
similar to the techniques performed in their daily clinical activities, with the purpose of reproducing their movements for the proposed analysis (Fig. 1).

\section{Rotary Instrumentation}

The chemomechanical preparation of the maxillary right second molar was carried out using the free tip preparation technique (9). After removing all remaining carious dentin
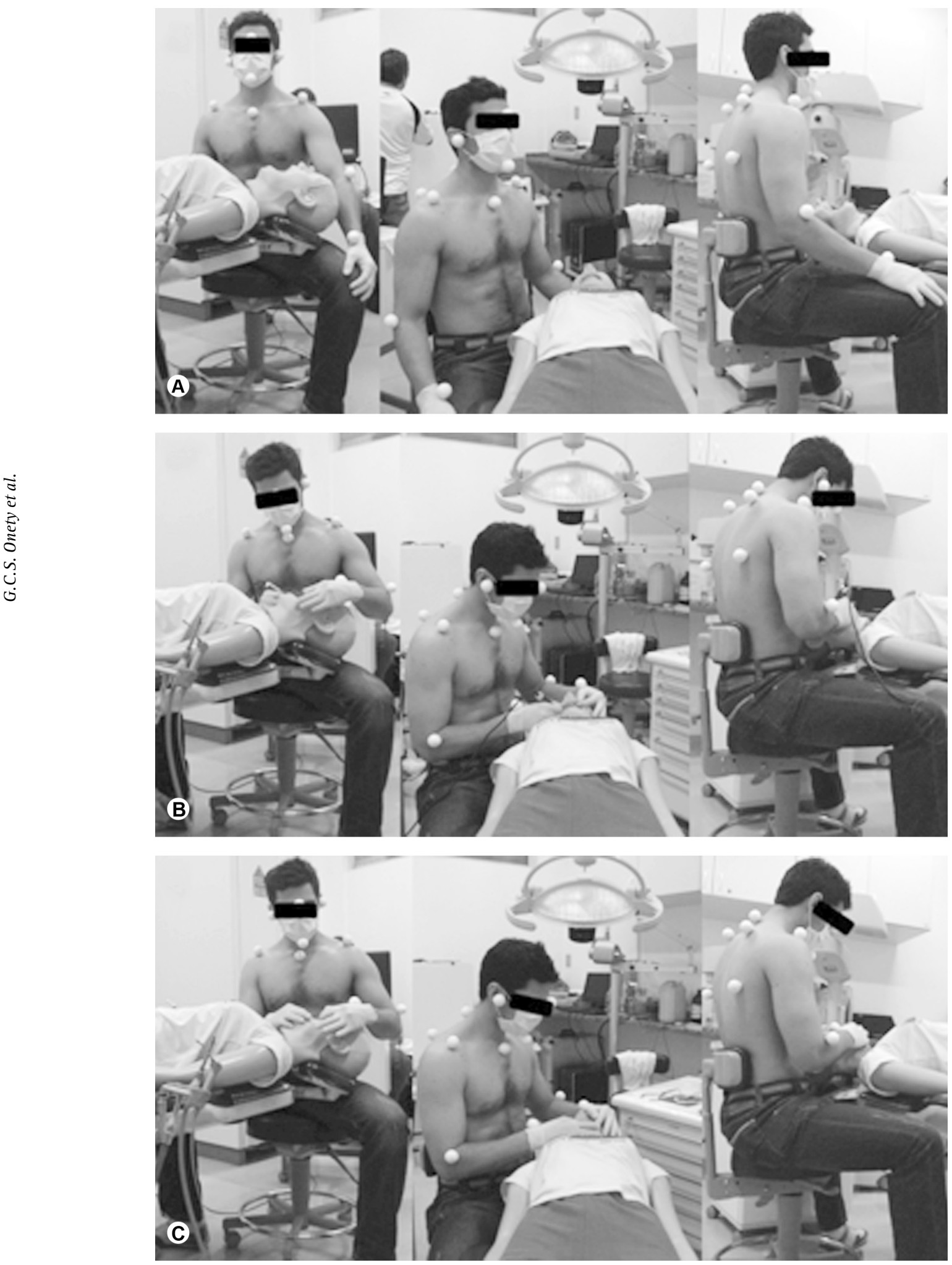

Figure 1. Evaluation of professional endodontist at rest (A), during rotary (B) and manual instrumentation (C). 
and defective restorations and irrigating the crown cavity with $1 \% \mathrm{NaOCl}$, the cervical region of the root orifices was prepared using a $\mathrm{K} 3 \mathrm{Ni}-\mathrm{Ti}$ instrument with a 0.06 taper and a 0.25 tip diameter (SybronEndo, Orange, CA, USA) with the speed set at $300 \mathrm{rpm}$. The instrument was advanced until it reached the working length (approximately $2 / 3$ of the total length at this stage) performing an up-and-down movement following its long axis. The procedure was carried out under copious irrigation with $1 \% \mathrm{NaOCl}$ with suction.

Then, a Ni-Ti instrument with a greater taper $(0.08$, 0.10 or 0.12 ) and a 0.25 tip diameter was used under copious irrigation and suction until it reached $2 / 3$ of the total length. For instrumentation of the apical portion of the root canal, smaller taper (0.02 and 0.04) instruments were used with a tip diameter $\left(D_{1}\right)$ based on the Anatomical Diameter (AD) of the orifice that was required to reach the working length. The preparation was followed by the use of rotary instruments with tip diameters $\left(D_{1}\right)$ four numbers greater than those used previously to establish a Surgical Diameter (SD) of approximately $200 \mu \mathrm{m}$ of dilation in this region. The tooth was constantly irrigated with $1 \% \mathrm{NaOCl}$ in conjunction with aspiration during this stage.

After the procedure was completed, a cotton pellet was placed inside the canal, and the cavity was sealed with Coltosol cement as a provisional sealant material (Vigodent). Following the use of the rotary instrumentation, the professional was allowed to rest before beginning the manual instrumentation of the maxillary right first molar.

\section{Manual Instrumentation}

To perform the manual instrumentation of the maxillary right first molar, was used a technique that is either known as Oregon technique, crown-down pressureless technique or crown-down technique. This technique requires more time than rotary instrumentation and involves no trauma to the apical segment. Initially, after removal of the temporary seal of dentin with a curette and irrigation of the canal with

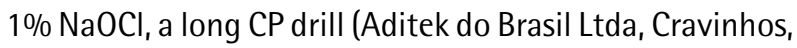
$\mathrm{SP}, \mathrm{Brazil})$ was used to widen the canal in the coronal third. Then, a manual type $\mathrm{K} I 0$ file (Maillefer) with a diameter equivalent to the mouth of the canal was selected. The instruments were introduced into the root canal with a clockwise $1 / 4$ rotation and then a counterclockwise $1 / 4$ rotation with adequate apical pressure. This procedure was repeated with instruments of decreasing diameter until an endodontic instrument number 10, 15 or 20 reached the working length that had been previously obtained.

The instrumentation was re-introduced into the canal by a file with a greater diameter than the initial file to reach the working length with a larger diameter endodontic file. The diameter was successively increased until the apical area was prepared with a file $0.30,0.35$ or $0.40 \mathrm{~mm}$ in diameter, dilating the diameter of the canal orifice to approximately $200 \mu \mathrm{m}$. Irrigation with $1 \% \mathrm{NaOCl}$ was performed every time the files were exchanged, and the canal chamber was always kept full of liquid. After the chemomechanical preparation was completed, a cotton pellet was placed inside the canal to seal the cavity with Coltosol.

\section{Assessments During the Chemomechanical Preparation \\ Electromyography Analysis}

Surface electromyography was performed using twelve channels of the portable MyoSystem-Brl apparatus (Datahominis Tecnologia, São Paulo, SP, Brazil), including eight channels for EMG (active and passive electrodes) and four auxiliary channels. A high-performance signal acquisition system and software to control the system and store and process the data were also used. The connectors have a DC input voltage of $\pm 12 \mathrm{~V} @ \pm 100 \mathrm{~mA}$, a CMRR (common-mode rejection ratio) of $112 \mathrm{~dB} @ 60 \mathrm{~dB}$, an input impedance for passive electrodes of 10 (10) Ohms/6 $\mathrm{pF}$, an input bias current for active electrodes of $\pm 2 \mathrm{nA}$, protection against overvoltage and band pass filters of 5 $\mathrm{Hz}$ to $5 \mathrm{kHz}$ to cancel out noise. Simple differential active electrodes were used (two $10 \mathrm{~mm}$ long and $1 \mathrm{~mm}$ wide silver chloride bars, separated by $10 \mathrm{~mm}$ ) and fixed in a resin capsule measuring $40 \times 20 \times 5 \mathrm{~mm}$.

Active electrodes with a signal gain of 20x were used to collect the electromyography parameters. The analog amplification of the signal was obtained with $124 x$ gain and analog filters of $2 \mathrm{kHz}$. The electrodes were positioned on the skin over the center of the following muscles: the longissimus region, the anterior and medium deltoids, the medium trapezium, the biceps brachii, the triceps brachii, the brachioradialis, and the short abductor of the thumb; the readings were collected unilaterally from the dominant limb of the endodontist, in this case, the right-hand side. The skin was cleaned with alcohol, and trichotomy was performed whenever necessary. The best points for the electromyography were determined following the standard international protocol SENIAM - Surface Electromyography for Non-Invasive Assessment of Muscles (10).

As a reference electrode (ground electrode), a stainless steel circular electrode $3 \mathrm{~cm}$ diameter was positioned over the skin of the right lateral malleolus. Then, the electromyographic points were marked on the skin at the center of the following muscles: the longissimus, the anterior and medium deltoid, the medium trapezium, the biceps brachii, the triceps brachii, the brachioradialis and the short thumb abductor. To normalize the data, an exercise called the crucifix or bridge was carried out with the endodontist sitting in the adjustable dental chair with both arms laterally open for $10 \mathrm{~s}$. After the initial reading, 
a timer was set and the professional was asked to begin the endodontic technique as recommended, starting with the rotary instrumentation and, after a rest period of ten minutes, ending with the manual instrumentation.

The environment was kept calm and quiet during the acquisition of electromyographic signals to eliminate problems during the kinematic and electromyographic analyses. Instructions and explanations were provided prior to each experiment. All examinations were carried out in the morning and afternoon between 08:00 am and 03:00 pm, eliminating any detectable circadian rhythm.

\section{Computed Videogrammetry}

In addition to EMG data collection, videogrammetry was used to investigate the dentist in his workplace. Three Brazil Fujifilm S1800 cameras were positioned at $90^{\circ}$ angles, forming a $270^{\circ}$ circuit around the endodontist. The tripods were placed in three delimiting planes (frontal, left and right sides). The images were collected based on the height patterns $(90 \mathrm{~cm}$ lateral and $140 \mathrm{~cm}$ frontal with respect to the floor) and the distance between the focal point of the image and the center point of the work (150 $\mathrm{cm})$. During the technical procedures, the endodontists double-sided tape.

In this study, five angles were used to visualize body structures (cervical flexion-extension, cervical inclination, cervicothoracic column, and contralateral shoulder adduction-abduction and flexion extension) at the following times in the work cycle: initial (0\%), 25\%, $50 \%, 75 \%$ and final (100\%). To standardize the video recording, an independent synchronization was performed by changing the environment light at the start of data collection.

The marked points were as follows: the manubrium of the sternum, the acromion, the lateral epicondyle of the humerus, the styloid process of the radius, the $7^{\text {th }}$ vertebra of cervical region, the lower and upper angles of the scapula, the auricular tragus, the mentual region and the glabella. The videos were recorded continuously during the applied instrumentation technique (rotary and manual). At the end of each collection, the videos were transferred to a portable computer and the important points were assessed using the Quintic Biomechanics 9.03 software (Quintic Consultancy Ltd., Sutton Coldfield, West Midlands, UK) in WMV format with a reduced speed. This analysis was carried out along the endodontist's working cycle time (initial, 25\%, 50\%, $75 \%$ and final). The working time periods were defined by mathematical values, and the frame-ware was obtained by the software based on the time scale of the working cycle.

\section{Ergonomic Scales}

Two methods were applied for the ergonomic analysis of the workplace: RULA and Couto's checklist using the Ergolandia 3.0 software (FBF sistemas, Belo Horizonte, $M G$, Brazil) together with the electromyographic and cinemetric evaluations. Both methods were applied while the endodontists were working. RULA generates a score list for specific postures and classifies the individual's activity posture using a scale from 1 to 7 , where the higher scores indicate a greater biomechanical risk of developing injuries.

Couto's checklist (5) reveals the ergonomic risks, which may vary according to the type of activity and the risks and situations observed in the workplace. The scores in this checklist can be interpreted as follows: items $A$ and $B$ indicate a lack of risk, item $C$ indicates the possibility of risk, item $\mathrm{D}$ indicates risk and item $\mathrm{E}$ indicates high risk.

\section{Computerized Biophotogrammetry}

In the present analysis, the endodontists were photographed to evaluate their postural patterns. The professionals wore light and comfortable clothes for the evaluation. Image standardization was achieved by positioning each participant on a three-dimensional level platform facing a simetrograph $(200 \times 100 \mathrm{~cm}$ and $10 \mathrm{~cm})$. Wall-to-ground leveling was performed before the position was set. The passive markers were positioned (polystyrene balls $25 \mathrm{~mm}$ diameter) on the following body segments: the manubrium of the sternum, the acromion, the lateral epicondyle of the humerus, the styloid process of the radius, the $7^{\text {th }}$ vertebra of the cervical region, the lower and upper scapula angle and the $4^{\text {th }}$ vertebra of the lumbar region. A Brazil Kodak p880 8.5 Mp camera was positioned on a tripod ( $0.90 \mathrm{~m}$ high) with a $3 \mathrm{~m}$ distance between the camera focal lens and the center of the body, which was marked with adhesive tape for further evaluation. The images were taken by the same person without zoom in three different planes: anterior, lateral and posterior.

\section{Data Analysis}

The Myosystem- $\mathrm{Br} 1$ version 3.5.6. was used to collect the EMG signals. After digitation, the signals were filtered by a 0.20 to $2 \mathrm{kHz}$ band-pass filter and sampled by a 12-b $\mathrm{A} / \mathrm{D}$ converter with an acquisition frequency of $2 \mathrm{kHz}$. Signal windowing was used for data analysis, also referred to as the RMS EMG technique. This technique consists of generating a pre-determined temporal estimation of the collected signal. Five temporal estimations were conducted lasting $120 \mathrm{~s}$ each. To determine these time periods, all the muscle activities of the endodontist were summed 
to calculate the onset time interval and the initial, 25\%, $50 \%, 75 \%$ and final times of each technique. Subsequently, electromyographic signals were obtained while performing the instrumentation techniques used in routine clinical practice. The endodontists used the rotary technique for second molar took a $10 \mathrm{~min}$ rest period, and then performed the manual procedure for first molar without removing the electrodes. This methodology avoided the introduction of bias in the electromyography data due to electrode reapplication. Five time periods were selected to analyze the electromyographic values: initial, 25\%,50\%, 75\% and final, with each interval lasting $120 \mathrm{~s}$. The biceps brachii, triceps brachii, longissimus and medium trapezium muscles exhibited higher electromyographic values when the rotary technique was used. These results indicate the aggressive strain that affects endodontists who use vibratory devices.

Videogrammetry was used to analyze the biomechanical demands, diagnose the posture and provide suggestions for improvement to enable the work to be carried out safely and comfortably (7). This study used five angle axes to visualize body structures (cervical flexionextension, cervical inclination, cervicothoracic column, and contralateral shoulder adduction-abduction and flexion extension) according to the period of time in the work cycle (initial, 25\%, 50\%, 75\% and final). As a criterion for the standardization of the video recording, an independent synchronization was performed by changing the environment light at the start of data collection. The videos were analyzed according to the images taken by each camera: the left lateral camera (abduction-adduction of the contra-lateral shoulder and cervical tilt), the frontal camera (flexion-extension of the elbow and flexion- extension of cervical) and the right lateral camera (cervico-thoracic column).

Free closed angles were drawn according to the endodonist's clinical condition. A protocol set a normalization size band of 100 frames to obtain the exact time of the performed activity. Images were obtained in three planes to quantify the endodontist's activity in a spatial analysis. Three independent cameras were positioned in the workplace to record the videos while the two instrumentation techniques were used. To minimize possible bias in the results, several standardization criteria were established for these recordings, including the ground marking; tripod height; positioning of the endodontist at 9 or $11 \mathrm{~h}$ to reduce the variation in the adjustable chair during the procedure; and the distance of the focal point of the lens to the endodontist. The following formula was used to calculate the mathematical expressions used to identify the work periods: (video full time - initial stage post sync) $x 0.25,0.50$ or 0.75 . The final work period coincided with the conclusion of the procedures.
Depending on the RULA score, the following interventions are required: level 2 - posture should be investigated and corrected; level 3 - posture needs to be investigated and changed in the near future to prevent an injury; level 4 - posture needs to be changed immediately to prevent an injury. Level 2 indicates an acceptable posture. Couto's checklist classifies risk using biomechanical criteria: A: the absence of significant factors (0 to 3 points); B: biomechanical factors of low significance (4 to 6 points); C: biomechanical factors of moderate significance (7 to 9 points); D: significant biomechanical factors (10 to 14 points); and E: highly significant biomechanical factors (above 15 points).

The biophotogrammetry images were processed by ImageJ software (National Institutes of Health, Bethesda, $M D$, USA) version $1.40 \mathrm{~g}$, with prior calibration by the Set Scale command using the simetrograph. Closed and free angles were subsequently marked in the following clinical conditions: shoulder elevation and abduction, elbow flexion and scapula rotation of bilateral regions.

\section{Results}

The standardized electromyographic data, videogrammetry data, biophotogrammetry data and ergonomic scales were tabulated and statistically analyzed using SPSS version 17.0 for Windows (SPSS, Inc., Chicago, IL, USA). A descriptive analysis was carried out (means, standard deviations, maximum and minimum values) for each variable and these values were compared by an independent t-test.

\section{Surface Electromyography}

In the initial period of the work, higher normalized electromyographic values were observed during the rotary technique in most muscles, except for the anterior deltoid and the short abductor of the thumb. At 25\% of the procedure time, higher standardized electromyographic values were obtained while using the rotary technique in the biceps brachii, triceps brachii, brachioradialis and longissimus muscles, but these differences were not significant. The anterior and medium deltoid, medium trapezium and short thumb abductor muscles showed higher electromyographic activities while the manual technique was performed. At 50\% of the procedure time, the biceps brachii, triceps brachii and longissimus muscles exhibited higher normalized electromyographic values during the rotary technique, but these differences were not significant. The anterior, medium deltoid, brachioradialis, medium trapezium and short thumb abductor muscles showed higher normalized electromyographic activity during the performance of the manual technique. At 75\% of the procedure time, the biceps brachii, triceps brachii and 
longissimus muscles exhibited higher electromyographic values during the performance of the rotary technique, while the anterior, medium deltoid, brachioradialis, medium trapezium and thumb short abductor muscles showed greater electromyographic activity during the performance of the manual technique. The medium trapezium muscle had similar standardized electromyographic values in both techniques. At the end of the procedure, the biceps brachii, triceps brachii, medium trapezium and longissimus muscles exhibited higher electromyographic values during the rotary technique.

The anterior, medium deltoid, brachioradialis, medium trapezium and short thumb abductor muscles showed greater electromyographic activity during the performance of the manual technique. At $25 \%$ of the procedure time, the data for the short thumb abductor muscle were significantly different $(p \leq 0.05)$ between both techniques. At $75 \%$ of the procedure time, significant differences between procedures were observed for the anterior $(p \leq 0.05)$ and medium ( $p \leq 0.01)$ deltoid and short thumb abductor $(p \leq 0.01)$ muscles. At the end of the procedure $(100 \%)$, the only significant difference $(p \leq 0.05)$ was for the short thumb abductor muscle (Table 1).

\section{Computerized Videogrammetry}

In the clinical condition of flexion-extension of the cervical spine, a prevalence of neck flexion in the work cycle was initially observed during the performance of the manual technique. Neck flexion was more prevalent at 50\% elapsed time during the rotary technique than at $25 \%$ or $75 \%$. During the cervical tilt, in the initial work cycle and at
$25 \%$, greater cervical inclination to the right was observed during the manual technique. In the rotary technique, a small cervical inclination to the right was observed at 75\% and at the end of the working cycle. The cervico-thoracic column showed that endodontists presented a thoracic hyperkyphosis throughout the work cycle for the rotary technique, regardless of the instrumentation period, which was not observed during the performance of the manual technique.

For the clinical condition of flexion-extension of the elbow, the rotary technique resulted in a higher angle load (elbow semi-flexion) than the manual technique during the work cycle, except in the initial phase of instrumentation. In the clinical condition of adduction-abduction of the contralateral shoulder, the rotary technique presented greater shoulder abduction during the initial period and at 25 and 75\% of the workcycle, while the normality values remained the same at the other clinical time points. At the $50 \%$ and $75 \%$ time points, statistically significant values $(p \leq 0.05)$ were obtained with both techniques (Table 2).

\section{Ergonomic Scales - Workplace Assessment}

The RULA assessment showed that the endodontists adopted incorrect postures at the workplace that could expose them to considerable ergonomic risks. This evaluation observed average RULA values of $6.9 \pm 0.73$. According to Couto's checklist, the endodontists had a moderate biomechanical risk of injury while at work, with average values of $8.1 \pm 0.37$. These results demonstrate that the professional endodontist requires moderate postural changes to reduce the risk of injury during the work cycle.

Table 1. Normalized electromyographic means $(\mu \mathrm{V})$ and standard error of anterior deltoid (AD), medium deltoid (MD), biceps braquii (BB), triceps braquii (TB), brachioradialis (BR), thumb short abductor (TSA), medium trapezium (MT) and longissimus ( $\mathrm{L}$ ) muscles in rotary and manual techniques for the clinical condition of routine work in final time ( $t$ test at $\mathrm{p} \leq 0.05$ )

\begin{tabular}{|c|c|c|c|c|c|c|c|c|c|}
\hline Time & Technique & $A D$ & MD & $\mathrm{BB}$ & $\mathrm{TB}$ & $\mathrm{BR}$ & TSA & $\mathrm{MT}$ & $\mathrm{L}$ \\
\hline \multirow{2}{*}{ Initial } & Rotary & $1.08 \pm 0.20$ & $0.20 \pm 0,01$ & $2.72 \pm 0.30$ & $2.50 \pm 1.74$ & $4.02 \pm 0.45$ & $33.59 \pm 3.39$ & $0.47 \pm 0.06$ & $2.99 \pm 0.32$ \\
\hline & Manual & $1.20 \pm 0.26$ & $0,18 \pm 0.01$ & $2.62 \pm 0.31$ & $0.53 \pm 0.05$ & $3.73 \pm 0.38$ & $36.04 \pm 4.07$ & $0.44 \pm 0.06$ & $2.63 \pm 0.35$ \\
\hline \multirow{2}{*}{$25 \%$} & Rotary & $0.85 \pm 0.10$ & $0.23 \pm 0.02$ & $3.20 \pm 0.37$ & $2.15 \pm 1.32$ & $4.12 \pm 0.45$ & $32.83 \pm 3.66^{*}$ & $0.55 \pm 0.05$ & $2.89 \pm 0.31$ \\
\hline & Manual & $1.06 \pm 0.12$ & $0.25 \pm 0.02$ & $2.80 \pm 0.37$ & $0.57 \pm 0.05$ & $3.72 \pm 0.38$ & $47.90 \pm 5.02^{*}$ & $0.56 \pm 0.13$ & $2.61 \pm 0.40$ \\
\hline \multirow{2}{*}{$50 \%$} & Rotary & $0.84 \pm 0.11$ & $0.21 \pm 0.02$ & $3.30 \pm 0.28$ & $1.90 \pm 1.08$ & $4.13 \pm 0.35$ & $29.09 \pm 3.12^{* *}$ & $0.49 \pm 0.05$ & $2.64 \pm 0.28$ \\
\hline & Manual & $1.12 \pm 0.16$ & $0.27 \pm 0.02$ & $2.97 \pm 0.39$ & $0.58 \pm 0.04$ & $4.17 \pm 0.45$ & $49.34 \pm 5.45^{* *}$ & $0.71 \pm 0.25$ & $2.40 \pm 0.29$ \\
\hline \multirow{2}{*}{$75 \%$} & Rotary & $0.67 \pm 0.07^{*}$ & $0.18 \pm 0.16^{* *}$ & $3.34 \pm 0.36$ & $2.58 \pm 1.55$ & $4.14 \pm 0.39$ & $25.60 \pm 2.77^{* * *}$ & $0.47 \pm 0.04$ & $3.95 \pm 1.26$ \\
\hline & Manual & $0.99 \pm 0.12^{*}$ & $0.27 \pm 0.02^{* * *}$ & $3.06 \pm 0.42$ & $0.58 \pm 0.05$ & $4.16 \pm 0.49$ & $48.46 \pm 4.98^{* * *}$ & $0.47 \pm 0.06$ & $2.65 \pm 0.39$ \\
\hline \multirow{2}{*}{ Final } & Rotary & $0.86 \pm 0.08$ & $0.17 \pm 0.02$ & $2.98 \pm 0.31$ & $1.94 \pm 1.25$ & $3.79 \pm 0.42$ & $25.96 \pm 3.15^{*}$ & $0.62 \pm 0.17$ & $3.59 \pm 1.17$ \\
\hline & Manual & $0.91 \pm 0.10$ & $0.20 \pm 0.20$ & $2.86 \pm 0.37$ & $0.59 \pm 0.05$ & $3.97 \pm 0.56$ & $36.47 \pm 3.94^{*}$ & $0.46 \pm 0.06$ & $2.37 \pm 0.33$ \\
\hline
\end{tabular}

** Significant with respect to initial (0\%), 25\%, 50\%, 75\% and final (100\%) p $\leq 0.01$. * Significant with respect to initial (0\%), 25\%, 50\%, 75\% and final $(100 \%) \mathrm{p} \leq 0.05$. 


\section{Computerized Biophotogrammetry}

The results of computerized biophotogrammetry showed that the left shoulder (LS) was slightly higher than the right during whole work cycle in both techniques. As for the elbow flexion (EF) on the right side, the load angle (elbow static semi-flexion) was higher than the one on the left. Similar shoulder abduction (lateral elevation) values were observed between the right and left sides. For the clinical condition of scapula rotation, the right side presented greater external rotation. These results were only significant $(p \leq 0.05)$ for elbow flexion (Table 3 ).

\section{Discussion}

This investigation used the electromyographic technique to assess the muscular changes experienced by endodontists while performing two different endodontic methods (rotary and manual), which were compared at different periods: initial, 25\%, 50\%, 75\% and final.

Vibratory equipment, such as used in rotary systems, operates by generating a vibratory or acceleration energy. A percentage of this energy is then transferred to the body of the operator and transformed into mechanical energy. A higher level of muscle activity was observed when endodontists used the rotary technique, leading to a significant requirement for stabilization of the upper limb due to the release of energy (11). A greater activation of the synergistic and stabilizer muscles in the shoulder was detected, demonstrating that the use of the rotary system influenced the muscle activity in the elbow, shoulder and spine, increasing the stability of these regions due to functional demands.
The function of the shoulder is to allow for a greater magnitude of hand movement with respect to workspace. The shoulder region can accomplish extensive movement in three axes: anterior-posterior, side-by-side and rotational, as result of a small amount of static stabilizer. The long portions of the biceps brachii and triceps brachii muscles are the glenohumeral and synergistic stabilizers of the shoulder movements. A greater need for joint stabilization was observed after the rotary system was used for a certain time. The longissimus muscle indirectly affects the stability of the shoulder joint, as the involuntary contractions of this muscle stabilize the column articulation and maintain the body posture (12)

At the end of the work cycle for both techniques, the medium trapezium muscle had higher activity during the rotary technique; however, the endodontists had already exhibited some postural changes. This condition may be the result of muscle fatigue, since the trapezium muscle acts as a stabilizer during scapula rotation, which allows for greater freedom and support of the shoulder movements and minimizes the muscle compensation in this region (13).

The repetition of movements during endodontic treatments leads to incorrect postures in the arm and trunk, and a lack of maintenance of body alignment, generating occupational pathologies as a result of greater muscular overload in the region described above. There was also a substantial increase in the activity of the short thumb abductor in the rotary technique, compared with manual technique, mainly due to the pen-grasp movement that is performed during the use of a rotary hand piece and/or manual instrumentation; a higher level of muscular activity

Table 2. Videogrammetry mean values and standard error of angular axis of cervical flexion-extension (CFE), cervical inclination (CI), cervico-thoracic column (CTC), elbow flexion-extension (EFE) and shoulder adduction-abduction (SAA) in rotary and manual techniques for the comparative analysis between the techniques during the work cycle of endodontists

\begin{tabular}{lcccccc}
\hline $\begin{array}{l}\text { Temporal } \\
\text { Analysis }\end{array}$ & Techniques & CFE & Cl & CTC & EFE & SAA \\
\hline \multirow{2}{*}{ Initial } & Rotary & $83.92 \pm 1.78$ & $103.30 \pm 4.42$ & $148.48 \pm 2.29$ & $104.74 \pm 2.99$ & $91.70 \pm 1.44$ \\
& Manual & $81.94 \pm 2.13$ & $100.78 \pm 3.80$ & $149.97 \pm 2.57$ & $102.62 \pm 3.29$ & $90.81 \pm 1.63$ \\
& Rotary & $103.78 \pm 5.06$ & $52.29 \pm 6.55$ & $136.13 \pm 2.52$ & $51.95 \pm 2.91$ & $92.78 \pm 2.98$ \\
$25 \%$ & Manual & $108.77 \pm 4.90$ & $46.77 \pm 4.70$ & $137.71 \pm 2.05$ & $58.52 \pm 2.47$ & $88.62 \pm 2.13$ \\
& Rotary & $106.36 \pm 4.96$ & $48.90 \pm 5.50$ & $139.49 \pm 2.93$ & $52.13 \pm 2.34^{* *}$ & $89.11 \pm 4.67$ \\
$50 \%$ & Manual & $102.81 \pm 4.16$ & $48.89 \pm 4.65$ & $141.10 \pm 2.44$ & $64.36 \pm 3.63^{* *}$ & $89.92 \pm 2.89$ \\
$75 \%$ & Rotary & $103.49 \pm 6.41$ & $47.51 \pm 6.01$ & $138.59 \pm 2.49$ & $49.93 \pm 2.54^{* *}$ & $92.73 \pm 2.97$ \\
& Manual & $104.75 \pm 5.39$ & $48.22 \pm 4.31$ & $140.39 \pm 1.55$ & $62.46 \pm 4.22^{* *}$ & $90.79 \pm 3.02$ \\
Final & Rotary & $106.50 \pm 5.41$ & $46.37 \pm 5.37$ & $137.53 \pm 3.09$ & $49.93 \pm 2.06$ & $99.76 \pm 3.67$ \\
& Manual & $106.61 \pm 5.12$ & $47.67 \pm 5.41$ & $139.33 \pm 1.70$ & $51.16 \pm 2.52$ & $99.48 \pm 3.54$ \\
\hline
\end{tabular}

** Statistically significant with respect to initial (0\%), 25\%, 50\%, 75\% and final (100\%) $p \leq 0.01$. 
was also observed in the wrist (14).

In the manual technique, greater activity was observed in the anterior and medium deltoid, as well as in the short thumb abductor muscles. This technique requires a $1 / 4$ rotation and a $1 / 2$ turn of the file inside the root canal, requiring greater muscle activation of the short thumb abductor $(8,15)$. The deltoid muscles (anterior and medium) were more active during the manual technique throughout the whole work cycle. This occurred because these muscles are influenced by fine coordinating movements, such as the highly precise movements performed by the endodontist's hands, leading to muscle adaptations throughout the forearm and arm to stabilize the glenohumeral joint. These results showed the influence of the deltoid muscle on shoulder stability, acting with the rotator cuff and the long head of the biceps to stabilize the glenohumeral joint. However, these results indicated that the deltoid muscles do not influence fine coordination, which is governed only by the trapezium muscle, which contributes to shoulder stabilization (16).

The brachioradialis muscles exhibited their highest electromyographic values at the beginning of the data collection period during the rotary technique. This is due $\therefore$ to the attempt to increase the stability of the ulno-humeral joint. These findings demonstrate that the brachioradialis muscle is important for elbow stability (17). Increased muscle activity was observed in the brachioradialis muscle during the manual technique. This can be explained by the decrease in the activity of the deltoid muscles, which generates a compensatory muscular mechanism in which a decrease in the shoulder stability stimulates the synergistic muscles (biceps and triceps brachii), triggering an increase in the activity of the brachioradialis to better stabilize the

Table 3. Biophotogrammetry and standard error of clinical condition right and left shoulder elevation, right and left elbow semi-flexion, right and left shoulder abduction and right and left scapula rotation in endodontists ( $t$ test at $\mathrm{p} \leq 0.05$ )

\begin{tabular}{lccc}
\hline Region & $\mathrm{P}$ & Mean & Standard Error \\
\hline Right shoulder elevation & & 99.34 & \pm 0.97 \\
Left shoulder elevation & $0.17^{\mathrm{ns}}$ & 101.10 & \pm 0.83 \\
Right elbow semi-flexion & & 158.23 & \pm 1.55 \\
Left elbow semi-flexion & $0.01^{* * *}$ & 163.80 & \pm 1.57 \\
Right shoulder abduction & & 100.33 & \pm 1.05 \\
Left shoulder abduction & $0.72^{\text {ns }}$ & 99.77 & \pm 1.21 \\
Right scapula rotation & & 126.75 & \pm 2.69 \\
Left scapula rotation & $0.11^{\text {ns }}$ & 121.35 & \pm 2.01 \\
\hline
\end{tabular}

**Statistically significant with respect to the right and left side $p \leq 0.01$.

ns Not significant

516 elbow (Table 1).

The results related to the flexion-extension of the cervical spine showed a high degree of neck flexion in the manual technique at the beginning of the working cycle and at $50 \%$ of the procedure time. Likewise, there was a prevalence of neck flexion in the rotary technique at $75 \%$ and $25 \%$ of the working cycle. These results demonstrate the prevalence of cervical flexion during the work routine. The clinical routines of dentists include several procedures during which these professionals adopt and maintain positions that can affect the integrity of their skeletal muscle system (18). These positions include arm lifting without support and flexing and rotating the cervical spine, which were also recorded in this study. There is a high prevalence of symptoms in the neck and shoulder as well as the lumbar region during clinical practice.

For the clinical condition of cervical inclination, the manual procedure only resulted in greater cervical inclination to the right in the initial period of the work cycle and at 25\%. During the rotary procedure, a low level of cervical inclination to the right was observed at $75 \%$ of the procedure time and at the end of the work cycle. The endodontists will seek the best posture for the use of the instrumentation, especially during the manual technique. Proper posture at the workstation must be adopted so that the neck remains in a neutral position, without inclination, flexion-extension and/or rotation, keeping the visual field at a precise level so that it does not affect the skeletal muscle system, as both techniques require accurate visual focus for effective performance. The visual field is set according to the placement of instrumentation equipment, regardless of the technique to be employed. However, appropriate adjustment of the clinician's chair during instrumentation or body positioning could reduce cervical pain (19).

The postural results of flexion-extension and cervical inclination showed that the manual technique presented a greater kinesio-function in the biomechanical aspects of posture, but this difference was not significant for all aspects. These data are consistent with the postural demands of the technique. The dentist's field of vision can be impaired by the need of adapting to the limitations of the oral cavity opening, as well as by the morphology of the teeth, poor access and lack of a direct view. The endodontist must perform the procedure without resting the arms on the patient while maintaining local hygiene. The endodontist maintains the neck and head in a flexed position most of the time, which may cause pain in these regions (20).

In the cervico-thoracic spine, the rotary technique caused a greater thoracic hyperkyphosis in the dentist than the manual technique throughout the work cycle regardless of the used instrumentation, but this difference was not 
significant. The prevalence of thoracic hyperkyphosis observed in this study can be related to the adaptation of the clinician to the vibratory equipment. These findings are in agreement with a report showing that the most frequent postural disorders among dentists are scoliosis and hyperlordosis, including thoracic hyperkyphosis (21).

With regard to the flexion-extension of the elbow, the rotary technique led to a greater loading angle (elbow semi-flexion) than the manual technique throughout the work cycle with the exception of the initial phase of instrumentation. In this study, it was found that while handling the instrumentation, the rotary technique presented a greater functional demand than the manual technique, demonstrating that this technique could lead to greater postural and functional changes in the skeletal muscle system (22) (Table 2).

For the clinical condition of left shoulder adductionabduction, the rotary technique presented the highest values during the initial, 25\%,50\% and 75\% work cycles, but the differences between techniques were not significant. During the handling of the counter-angle handpiece, some postural adaptations of the arm occurred. In addition, the pattern of postural adjustment exposed the endodontist to static efforts. Endodontists should minimize the flexion and abduction of the shoulders and support loads with their hands. Furthermore, they should reduce or eliminate tasks that require excessive use of manual force and should take longer rest periods. Repetitive and rapid movements of the arms or movements that require static efforts should be eliminated (23).

In this study, during the first 15 min of the endodontic procedure, the RULA and Couto's evaluations were carried out to analyze and classify the biomechanical factors that affect endodontists during their professional practice. The results obtained using RULA revealed higher index values for postural disorders during the rotary technique, with a score of 7, indicating the need for immediate application of ergonomic measures to prevent injuries.

According to Couto's checklist (5), the endodontists were classified as exhibiting biomechanical factors of moderate importance, indicating that injury is improbable but possible (C-biomechanical factor of moderate significance - score between 7 and 9 points), demonstrating that there is also an ergonomic disorder. These data revealed the importance of the muscles of the shoulder girdle and cervical spine for the biomechanical performance of the dentists, who reported fatigue and pain in these muscles during work (2). These data suggest that the dentists adopt compensatory postures at work resulting in moderate to high-risk levels. The results obtained in this study disagreed, in part, with those reported by David (4), who reported that the work postures at 9 and $11 \mathrm{~h}$ that are usually used by dentists could minimize postural disorders among dentists. In contrast, this study found a high ergonomic risk for WMSDs, even in these recommended work postures.

Professional activities force dentists to use their arms and adjacent structures during their work routines, where the same movement pattern is frequently repeated due to the clinical activity. Dentists adopt incorrect postures while using different instrumentation (24).

For the clinical condition of shoulder elevation, the left side appeared higher when compared with the right. These results revealed that the professionals tended to lean to the right to handle the instrumentation due to a postural change between the shoulders. Physical training programs should be used to achieve even elevation between the two shoulders, regardless of dominance, as this can enhance biomechanical characteristics and the postural pattern. For elbow flexion, the loading angle to the left side (elbow semi-flexion) was observed to be higher on the left side than on the right. These findings disagreed with those of Roman-Liu et al. (24), who found a normal pattern of elbow flexion in both semi-bodies of athletes. The prevalence of semi-flexion of the right elbow is most likely related to the fact that the endodontists were right-handed and placed a greater demand on the right upper limb, which exhibited structural adaptations during the activities (Table 3).

Similar shoulder abduction values were observed between the right and left sides, demonstrating that some body harmony was achieved without postural compensation. According to Pontillo et al. (25), there is a greater predisposition to alter the balance between mobility and stability when the shoulder is abducted. In this study, the shoulder abduction values possibly matched because the endodontists made few changes in the workplace. For the clinical condition of scapula rotation, the right-hand side presented a greater external rotation of the scapula. This may be related to the fact that the dentists were right-handed and imposed a greater demand on the right upper limb.

The scapula rotation is not a frequent condition. This condition is associated with muscle dysfunction of the anterior serratus, causing a displacement in the scapular complex. However, the results show that the clinical practices of endodontists can influence the shoulder muscular system, the elbow and the shoulder girdle, promoting an imbalance in the muscle activity of these structures and consequently leading to the onset of postural changes (17).

Based on these results, the variations in standardized electromyographic values during the handling of the rotary and manual techniques suggest that the actions of the longissimus, anterior and middle deltoid, medium trapezium, brachial biceps and triceps, brachioradialis 
and short thumb abductor muscles underwent muscle adaptations to enable more accurate functional movements. Computerized videogrammetry revealed that the rotary technique presented greater postural demands than the manual technique. This greater demand was also observed by the analysis of the work place and the biomechanics of the professional activity. The static analysis of body posture (biophotogrammetry) suggests that the clinical activity of the dentist may contribute to the postural imbalance of the upper body quadrant. Further studies are required to evaluate ergonomic equipment and techniques to minimize the postural and functional changes of endodontists during their working hours.

\section{Resumo}

Os fatores de risco posturais para cirurgiões dentistas incluem o acesso a visão no local de trabalho, frio, vibração, pressão mecânica nos tecidos, postura incorreta, alterações funcionais, requisitos cognitivos e fatores organizacionais e psicossociais relacionados com o trabalho. 0 objetivo é analisar a postura dos endodontistas no local de trabalho. Participaram dezoito endodontistas destros com idades entre as idades de 25 e 60 anos $(34 \pm 3)$. Nesta pesquisa foi utilizado a eletromiografia, cinemetria, escalas de ergonomia (do RULA e Couto checklist) e biofotogrametria para analisar a postura dos endodontistas durante o preparo quimico-mecânico do sistema de canais radiculares para primeiros e segundos molares superiores - direitos, utilizando a instrumentação rotatória e manual. As variações observadas nas atividades eletromiográficas durante a execução das técnicas rotatórias e manuais sugerem que as fibras da região dos músculos longuíssimo, deltóide anterior e médio, trapézio médio, biceps, triceps braquial, braquiorradial e músculos abdutores curtos do polegar passaram por adaptações para promover movimentos funcionais mais precisos. A cinemetria e biofotogrametria computadorizada mostraram que a técnica rotatória foi mais exigente da postura corporal do que a técnica manual. Em conclusão, os endodontistas estudados apresentaram distúrbios de postura, independentemente da técnica utilizada, rotatória ou manual.

\section{Acknowledgements}

Fundação de Amparo à Pesquisa de Amazonas (FAPEAM) for the financial support.

\section{References}

1. Rucker LM, Sunell S. Ergonomic risk factors associated with clinical dentistry. J Cal Dent Assoc 2002;30:139-148.

2. Pasternak Junior B, Sousa Neto MD, Dionísio VC, Pécora JD, Silva RG. Analysis of kinematic, kinetic and electromyographic patterns during root canal preparation with rotary and manual instruments. J Appl Oral Sci 2012;20:57-63.

3. Ahearn DJ, Sanders MJ, Turcotte C. Ergonomic design for dental offices. Work 2010;35:495-503.

4. David GC. Ergonomic methods for assessing exposure to risk factors for work-related musculoskeletal disorders. Occup Med 2005;55:190-199.

5. Albin TJ. Measuring the validity and reliability of ergonomic checklists. Work 2012;43:381-385.
6. Silva GP, Ferreira B, Gurian MB, Ribeiro EA, Verri ED, Nepomuceno $V R$, et al.. Association between the paraplegia and athletics sports in the upper limbs, posture and stomatognathic system. J Novel Physiot 2013;3:1-8.

7. Young S. Research for medical photographers: photographic measurement. J Audio Med Medic 2002;25:94-98.

8. Alencar AH, Dummer PM, Oliveira HC, Pécora JD, Estrela C. Procedural errors during root canal preparation using rotary NiTi instruments detected by periapical radiography and cone beam computed tomography. Braz Dent J 2010;21:543-549.

9. Sun $\mathrm{HH}$, Jin T, Yu Q, Chen FM. Biological approaches toward dental pulp regeneration by tissue engineering. J T Eng Reg Med 2011;5:1-16.

10. Hermens HJ, Freriks B, Disselhorst-Klug C, Rau G. Development of recommendations for SEMG sensors and sensor placement procedures. J Elect Kinesio 2000;10:361-374.

11. Lins CCS, Silva EMVM, Lima GA, Menezes SEAC, Travassos RMC. Operating microscope in endodontics: A systematic review. Sci Res 2013;3A:1-5

12. Dworak LB, Murawa M, Owsian M, Maczyuski J, Kabaciuski J, Rzepnicka A. Three point crutch gait from the perspective of biomechanics and kinesiology. State of knowledge and idea behind the research. Chi Narz Ruc Ortop Pols 2011;76:305-312.

13. Siéssere $S$, Sousa $L G$, Lima NA, Semprini M, Vasconcelos PB, Watanabe PCA, et al.. Electromyographic activity of masticatory muscles in women with osteoporosis. Braz. Dent J 2009;20:237-242.

14. Ahmad IA, Al-Jadaa A. Three root canals in the mesiobuccal root of maxillary molars: Case reports and literature review. J Endod 2014;40:2087-2094.

15. Savani GM, Sabbah W, Sedgley CM, Whitten B. Current trends in endodontic treatment by general dental practitioners: report of a United States national survey. J Endod 2014;40:618-624.

16. Prentice WE, Voight ML. Techniques in musculoskeletal rehabilitation. Porto Alegre: Artmed, 2003.

17. Van Roy $P$, Baeyens JP, Fauvart $D$, Lanssiers R, Clarijs JP. Arthrokinematics of the elbow: study of the carrying angle. Erg 2005:15:48:1645-1656.

18. Farag $\mathrm{AA}$, Eid $\mathrm{A}$. Video reconstructions in dentistry. Orth Cranio Res 2003;6:108-116.

19. Kimmel K. The workplace of the dentist. Problems of organization and equipment. Dental Echo 1976;46:10-15

20. Zarra T, Lambrianidis T. Musculoskeletal disorders amongst Greek endodontists: a national questionnaire survey. Int Endod J 2014;47:791-801

21. Alexopoulos EC, Stathi IC, Charizani F. Prevalence of musculoskeletal disorders in dentists. BMC Musc Dis 2004;5:1-8.

22. Dick FD, Graveling RA, Munro W, Walker-Bone K. Guideline Development Group. Workplace management of upper limb disorders: a systematic review. Occup Med 2011;61:19-25.

23. Riva $G$, Mantovani $G$. The ergonomics of virtual reality: human factors in developing clinical-oriented virtual environments. S H Tech Inform 1999:62:278-284.

24. Liu P, McGrath C, Cheung GS. Improvement in oral health-related quality of life after endodontic treatment: a prospective longitudinal study. J Endod 2014;40:805-810

25. Pontillo M, Orishimo KF, Kremenic IJ, McHugh MP, Mullaney MJ, Tyler TF. Shoulder musculature activity and stabilization during upper extremity weight-bearing activities. N Am J Sp Phys Ther 2007;2:90-96.

Received January 23, 2014 Accepted September 28, 2014 